\title{
The Effect of Loudness Imbalance between Electrodes in Cochlear Implant Users
}

\author{
P.W. Dawson, M. Skok, and G.M. Clark
}

\begin{abstract}
Objective: The aim was to determine the effect of loudness imbalance between electrodes in patients using the 22-electrode cochlear implant (Cochlear Pty Ltd). It was hypothesized that speech perception scores would be greater when the loudness of electrodes was balanced at the comfort (C) levels than when the $C$ levels were unbalanced.
\end{abstract}

Design: Ten adult patients received a monosyllabic word test (CNC words) in quiet and a sentence test (CUNY sentences) in noise under two conditions: with $C$ levels balanced for equal loudness and with unbalanced $C$ levels.

Results: When the $\mathrm{C}$ levels across electrodes were pseudo-randomly unbalanced by 0 to $\pm 20 \%$ of the electrodes' dynamic ranges (20\% unbalancing), 6 of the 10 subjects showed a significant drop in sentence perception scores. Of these patients, none had a significant decrease in perception when the degree of unbalancing was halved. Of the four patients who showed no change with $20 \%$ unbalancing, three revealed a significant decline in sentence perception when the degree of unbalancing was doubled. There also were significant group effects for phonemes on the word test as well as for sentences in noise for the $20 \%$ unbalancing.

Conclusions: The implications for clinical practice are that it is important to balance the $C$ levels and that clinicians should be encouraged to refine methods for setting $C$ levels in very young children, who may be using unbalanced MAPs. Nevertheless, although most patients revealed a statistically significant drop in sentence perception with $20 \%$ imbalance of the $C$ levels, the changes in percentage scores often were only small.

(Ear \& Hearing 1997;18;156-165)

After implantation with a multichannel cochlear implant, time is spent fine tuning each of the electrodes for an individual client. An individualized speech processor program or MAP is obtained using specialized computer software. The softest levels that can be detected (i.e., thresholds or T levels) and the loudest levels that can be tolerated easily (i.e., comfort levels or C levels) are specified in this MAP.

Australian Bionic Ear and Hearing Research Institute (P.W.D., G.M.C.), 384-388 Albert St, East Melbourne 3002, Australia; and Cochlear Implant Clinic (M.S.), Royal Victorian Eye and Ear Hospital, 32 Gisborne St, East Melbourne 3002, Australia
Clinicians believe that it is important to balance the loudness of the electrodes at the $\mathrm{C}$ levels. This idea of balancing the loudness of the incoming speech signal for the various frequency bands is not a new one. Many hearing aid selection procedures aim to deliver all frequency bands of amplified speech at equal loudness and at a comfortable or preferred listening level (Berger, Hagberg, \& Rane, 1977; Byrne \& Tonnison, 1976; Skinner, Pascoe, Miller, \& Poelka, 1981). Otherwise, there is the possibility of one or a few frequency bands dominating the speech input and causing the aid user to turn down the volume control.

There is frequent anecdotal evidence from adult implant users that MAPs that are not balanced for loudness at the $C$ levels sound uneven and rough and have too much echo. Although there also is anecdotal evidence that an unbalanced MAP can adversely affect speech perception performance in adults using cochlear implants, there has been no published study to confirm this. It is unlikely, however, that adults using cochlear implants experience substantially unbalanced C levels. It is probable that for most adults the $\mathrm{C}$ levels are set with reasonable accuracy and reliability before the final balancing procedure, in which adjacent electrodes are balanced for equal loudness. In contrast, the $\mathrm{C}$ levels for many children younger than $5 \mathrm{yr}$ of age are likely to be far less accurate or reliable, given the children's inability to provide verbal judgments on what is comfortably loud. Also, the final balancing procedure often cannot be done with these children who typically have difficulty with same/different judgments. Hence, the MAPs for many children in this age range could be quite unbalanced.

One would think intuitively that an unbalanced MAP would affect speech perception adversely for a number of possible reasons. If the $\mathrm{C}$ levels on some electrodes are dropped substantially, some of the spectral information received on these electrodes may be too soft to be used effectively. In addition, unbalanced $\mathrm{C}$ levels would alter the natural amplitude cues of speech sounds, which provide important cues for phoneme identification. Manner and place of production differences between consonants are cued by amplitude differences as well as by frequency and timing differences (Blamey, Dowell, \& Clark, 1987; Van Tasell, Soli, Kirby, \& Widon, 1987). 
Intensity information also is important for vowel identification (Ptacek \& Koutstaal, 1977).

It is possible, however, that implant users may be able to tolerate substantial loudness imbalance between electrodes. The unbalanced MAP might sound less pleasant but have a minimal effect on speech perception. Alternatively, a small degree of imbalance may have a significant effect on speech perception and a minimal or significant effect on the pleasantness and acceptability of the sound. The relationship between the implantees' subjective responses to an unbalanced MAP and their speech perception performance is explored in the present study.

As stated earlier, an investigation into the unbalancing of $\mathrm{C}$ levels is relevant to the mapping of $\mathrm{C}$ levels in very young children, who usually are unable to indicate reliably when the sound is comfortably loud and to balance the loudness of electrodes at the $\mathrm{C}$ levels. The measurement of $\mathrm{C}$ levels (before loudness balancing of the electrodes) becomes more difficult as the age at implantation becomes increasingly younger. Clinicians often rely on behavioral observation audiometry to obtain loudness discomfort levels (LDLs) in a young child. Accepted behavioral responses might include an eye blink, sudden change in the child's play, facial grimace, and pulling the implant's transmitting coil off. The $\mathrm{C}$ levels are then usually set below the LDLs. Initially the $\mathrm{C}$ levels are set conservatively, and when the child gains experience they are raised (Staller, Beiter, \& Brimacombe, 1994). LDLs obtained in this way may be quite inaccurate at times. Objective techniques for setting $\mathrm{T}$ levels and $\mathrm{C}$ levels, such as electrically evoked stapedial reflex and electrically evoked auditory brain stem response, are being used more frequently now to confirm the behavioral levels (Battmer, Laszig, \& Lehnardt, 1990; Shallop, 1993; Shallop, VanDyke, Goin, \& Mischke, 1991; Spivak \& Chute, 1994). Such objective techniques still provide only approximate $\mathrm{C}$ level estimates. There is considerable variability in the methods used by clinicians to obtain $\mathrm{C}$ levels in children younger than $5 \mathrm{yr}$ of age.

This study aimed to simulate unbalanced MAPs with varying degrees of imbalance between electrodes. The type of imbalance that occurs when programming implants for children younger than 5 yr old is unknown. Random imbalance was chosen in this study because it was considered to be the worst case scenario. In contrast, systematic imbalance would be likely to have systematic effects, which can be compensated for. For example, fricative sounds may be too soft as a result of lower $C$ levels for the basal electrodes. The effect may be that the child fails to produce these sounds, unlike other implant users of a similar age and with similar implant experience. The observation of such an effect in the child's communication development may result in the audiologist's increasing the $\mathrm{C}$ levels on the basal electrodes.

The study addressed the question of how much imbalance can be tolerated before a significant decrease in speech perception occurs. The following specific hypotheses were investigated:

1. Perception scores for the balanced MAP will be significantly greater than those for the unbalanced MAPs.

2. Performance with the greatest degree of unbalancing will be worse than performance with the smallest degree of unbalancing.

\section{Methods}

Subjects - Ten patients implanted with the 22electrode cochlear prosthesis manufactured by Cochlear Pty Ltd (Clark et al., 1987) participated in the study. Criteria for inclusion were that all subjects had stable $\mathrm{T}$ and $\mathrm{C}$ levels in their MAPs and that phoneme perception performance for a word test in quiet was above $40 \%$. Table 1 summarizes patient details. The group includes two young adults implanted in adolescence who experienced progressive losses. Their performance after implantation has been similar to that of postlinguistically deafened adults. Furthermore, like the adults, they were able to balance the loudness of electrodes reliably soon after implantation. Four of the adults ( 7 through 10) were using the MSP processor with a Multipeak strategy encoding FO, F1, and F2, as well as three high frequency bands: 2.0 to $2.8 \mathrm{kHz}$ (Band 3), 2.8 to $4 \mathrm{kHz}$ (Band 4), and 4 to $6 \mathrm{kHz}$ (Band 5), onto three fixed basal electrode positions (Skinner et al., 1991). The remaining six adults ( 1 through 6 ) were using the Spectra 22 processor with the Speak speech coding strategy. With the Speak strategy, sound is analyzed with a maximum of 20 band-pass filters. An average of six electrodes are activated in each stimulation period. These electrodes correspond to the filters with the largest amplitudes (Skinner et al., 1994). Five patients were mapped in "bipolar + 1 " $(\mathrm{BP}+1)$ mode, in which electrodes are activated as pairs. The electrodes in each pair were separated by one electrode. For three patients using the BP + 2 mode of stimulation, electrodes in each pair were separated by two electrodes. One patient was mapped in common ground mode, in which the current flows from the electrode being stimulated to all other electrodes. For another patient, a pseudomonopolar mode was used, in which the current flows from the electrode being stimulated to the most basal electrode on the array. 
TABLE 1. Patient details.

\begin{tabular}{|c|c|c|c|c|c|c|c|c|c|}
\hline \multirow[b]{2}{*}{ Patient } & \multirow[b]{2}{*}{ Onset of Loss } & \multirow{2}{*}{$\begin{array}{l}\text { Duration of } \\
\text { Profound } \\
\text { Deafness }\end{array}$} & \multirow{2}{*}{$\begin{array}{c}\text { Age at } \\
\text { Implantation }\end{array}$} & \multirow{2}{*}{$\begin{array}{l}\text { Number of } \\
\text { Electrode } \\
\text { Channels }\end{array}$} & \multirow{2}{*}{$\begin{array}{l}\text { Mode of } \\
\text { Stimulation }\end{array}$} & \multirow{2}{*}{$\begin{array}{l}\text { Duration of } \\
\text { Implant } \\
\text { Use }\end{array}$} & \multicolumn{2}{|c|}{ Dynamic Range } & \multirow{2}{*}{$\begin{array}{l}\text { Signal to } \\
\text { Noise } \\
\text { Ratios }\end{array}$} \\
\hline & & & & & & & Min-Max & Mean & \\
\hline 1 & $\begin{array}{l}\text { Congenital } \\
\text { (progressive) }\end{array}$ & $1 \mathrm{yr}$ & $26 \mathrm{yr}, 4 \mathrm{mo}$ & 20 & $B P+1$ & $1 \mathrm{yr}$ & $30-56$ & 41 & +5 \\
\hline 2 & $18 \mathrm{yr}$ & $7 \mathrm{yr}$ & $47 \mathrm{yr}$ & 17 & $B P+1$ & $1 \mathrm{yr}, 1 \mathrm{mo}$ & $14-41$ & 33 & +8 \\
\hline 3 & $25 \mathrm{yr}$ & $11 \mathrm{mo}$ & $67 \mathrm{yr}, 7 \mathrm{mo}$ & 20 & $B P+1$ & $1 \mathrm{yr}, 3 \mathrm{mo}$ & $25-99$ & 62 & +8 \\
\hline 4 & $\begin{array}{c}\text { Congenital } \\
\text { (left ear) }\end{array}$ & $1 \mathrm{yr}$ & $33 \mathrm{yr}, 4 \mathrm{mo}$ & 12 & $\mathrm{BP}+2$ & $10 \mathrm{mo}$ & $50-90$ & 71 & +13 \\
\hline 5 & $16 \mathrm{yr}$ & $30 \mathrm{yr}$ & $50 \mathrm{yr}$ & 15 & $B P+1$ & $1 \mathrm{yr}$ & $37-70$ & 49 & +5 \\
\hline 6 & $7 \mathrm{yr}$ & $7 \mathrm{yr}$ & $34 \mathrm{yr}, 4 \mathrm{mo}$ & 15 & $\mathrm{BP}+2$ & $2 \mathrm{yr}, 4 \mathrm{mo}$ & $73-119$ & 98 & +5 \\
\hline 7 & $\begin{array}{l}\text { Congenital } \\
\text { (progressive) }\end{array}$ & $6 \mathrm{yr}, 10 \mathrm{mo}$ & $14 \mathrm{yr}, 8 \mathrm{mo}$ & 22 & $\mathrm{CG}$ & $6 \mathrm{yr}, 11 \mathrm{mo}$ & $46-98$ & 71 & +10 \\
\hline 8 & $\begin{array}{l}\text { Congenital } \\
\text { (progressive) }\end{array}$ & $10 \mathrm{yr}, 4 \mathrm{mo}$ & $18 \mathrm{yr}, 3 \mathrm{mo}$ & 20 & $\begin{array}{l}\text { Pseudo- } \\
\text { monopolar }\end{array}$ & $2 \mathrm{yr}, 3 \mathrm{mo}$ & $88-118$ & 103 & +15 \\
\hline 9 & $16 \mathrm{yr}$ & $5 \mathrm{yr}, 2 \mathrm{mo}$ & $42 \mathrm{yr}, 2 \mathrm{mo}$ & 17 & $B P+1$ & $1 \mathrm{yr}, 3 \mathrm{mo}$ & $21-50$ & 35 & +9 \\
\hline 10 & $21 \mathrm{yr}$ & $41 \mathrm{yr}, 9 \mathrm{mo}$ & $65 \mathrm{yr}, 9 \mathrm{mo}$ & 16 & $B P+2$ & $1 \mathrm{yr}, 4 \mathrm{mo}$ & $30-47$ & 38 & +15 \\
\hline
\end{tabular}

Note: Dynamic range data are in Cochlear Corporation stimulus fevel units.

For all patients, sound amplitude was coded using the stimulus level scale. $\mathrm{T}$ and $\mathrm{C}$ levels were expressed as stimulus levels. Over most of the stimulus level range (65 to 229), the current amplitude is constant at $1000 \mathrm{uA}$ and the pulse width (PW) changes exponentially. This increase in PW is associated with an increase in perceived loudness. In the lower and upper part of the stimulus level scale, the PW remains constant and the current amplitude increases exponentially (Skinner et al., 1991). The dynamic range (DR) in stimulus levels refers to the difference between the $\mathrm{T}$ and $\mathrm{C}$ levels. In Table 1 the mean DR across all electrodes and the minimum and maximum DRs are given.

Design - Patients were assessed in two conditions in each of a number of sessions (see Table 2).

1. Balanced condition (BC): A balanced MAP with electrodes balanced for loudness at the $\mathrm{C}$ levels.

The $\mathrm{C}$ levels in the patients' usual take-home MAPs were balanced for loudness in the first session. For some patients no changes were made to

\section{TABLE 2. Order of assessments.}

\begin{tabular}{|c|c|}
\hline Session 1 & $\begin{array}{l}\text { Balanced comfort levels for equal loudness in } \\
\text { patient's usual, take-home MAP. LDLs mea- } \\
\text { sured. }\end{array}$ \\
\hline Session 2 & $\begin{array}{l}\text { Assessed speech perception with balanced MAP } \\
\text { first and then unbalanced MAP } 1 \text { ( } 20 \% \text { unbal- } \\
\text { anced condition [UC]). }\end{array}$ \\
\hline Session 3 & $\begin{array}{l}\text { Assessed speech perception with unbalanced } \\
\text { MAP } 2 \text { first }(20 \% \text { UC) and then balanced } \\
\text { MAP. }\end{array}$ \\
\hline Session 4 & $\begin{array}{l}\text { Assessed speech perception with balanced MAP } \\
\text { first and then unbalanced MAP } 3(10 \% \text { or } \\
40 \% \text { UC). }\end{array}$ \\
\hline Session 5 & $\begin{array}{l}\text { Assessed speech perception with unbalanced } \\
\text { MAP } 4 \text { first }(10 \% \text { or } 40 \% \text { UC) and then bal- } \\
\text { anced MAP. }\end{array}$ \\
\hline
\end{tabular}

their usual MAP, whereas minimal changes occurred for other patients. Balancing involved sweeping across $\mathrm{C}$ levels for three electrodes at a time and asking the patient whether the electrodes sounded the same in loudness. An increment of two stimulus levels was used in making any necessary adjustments. At the beginning of subsequent sessions, patients were asked whether their take-home MAP (the balanced MAP) needed fine tuning. For all patients, the balanced MAP was stable for the duration of the study.

2. Unbalanced condition (UC):

C levels for each electrode were randomly unbalanced by up to $\pm 20 \%$ of the electrode's DR ( $20 \%$ unbalancing or $20 \%$ UC; see Fig. 1). A set of random numbers between -20 and +20 that summed to zero were generated and assigned to the patients' electrodes. A different set of random numbers was used

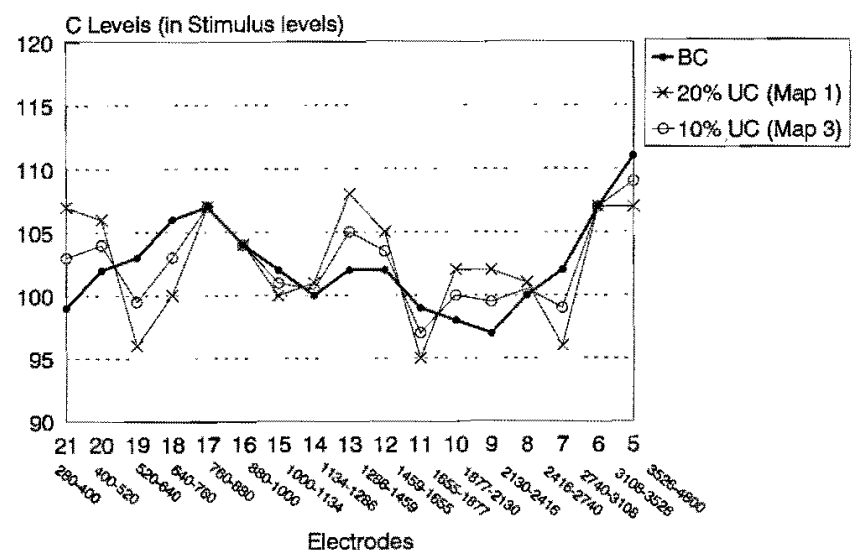

Figure 1. Comfort (C) levels for an individual patient (9), plotted as a function of the electrode number in the balanced condition $(\mathrm{BC})$ and in $\mathbf{2 0} \%$ and $10 \%$ unbalanced conditions (UC). The frequency boundaries represented by each electrode are provided. 
for each patient. The summation of the $\mathrm{C}$ level changes to zero minimized the possibility of MAPs becoming too soft or too loud. The authors acknowledge that an unbalanced MAP still could sound louder or softer than a balanced one despite this modification. For example, a MAP may sound louder when electrodes whose $\mathrm{C}$ levels were substantially raised were being more frequently stimulated.

Each patient was assessed with two different unbalanced MAPs ( 1 and 2) that were derived from different sets of random numbers. Each of these MAPs provided $20 \%$ unbalancing, but the changes to the $\mathrm{C}$ levels of individual electrodes differed in these two MAPs. Average magnitude deviations from the balanced MAP were, as expected, very similar for the two MAPs for each individual and close to $10 \%$ (the average value of 0 to $20 \%$ ). They ranged from $10 \%$ to $13 \%$. Each patient was assessed with more than one type of unbalanced MAP to increase the face validity of the study. An evaluation of the effect of loudness imbalance on speech perception based on results from only one randomly unbalanced MAP is likely to be less representative of what young children might experience in reality.

Unbalanced MAPs 3 and 4 were included to address Hypothesis 2 relating to the degree of unbalancing. MAP 3 was directly related to MAP 1 and MAP 4 was directly related to MAP 2 by a factor of two or one-half. If the average sentence perception performance with unbalanced MAPs 1 and 2 in the $20 \%$ UC was significantly worse than the performance in the $\mathrm{BC}$, the degree of unbalancing was halved to $10 \%$ in MAPs 3 and 4 (i.e., the set of random numbers that provided the percentage change for each electrode in MAPs 1 and 2 were halved in MAPs 3 and 4, respectively). Alternatively, if $20 \%$ unbalancing did not have a significant effect on sentence perception, the percentage change for each electrode was doubled in MAPs 3 and 4 (40\% unbalancing). Figure 1 shows the $C$ levels for an individual patient (Patient 9) plotted as a function of the electrode number in the $\mathrm{BC}$ and in a $20 \%$ $\mathrm{UC}$ and a $10 \% \mathrm{UC}$ for a particular randomization.

The unbalanced $\mathrm{C}$ levels did not exceed the patients' LDLs. In measuring an electrode's LDL, the patient was asked to indicate when the tone was uncomfortably loud and could not be tolerated for more than a few seconds. For patients 2, 3, 7, and 8 , LDLs on a few electrodes were slightly exceeded in the UC with the greatest amount of unbalancing. Adjustments were made to avoid this and to ensure that the $\mathrm{C}$ level changes summed to zero.

The order in which the $\mathrm{BC}$ and the UC were assessed was counterbalanced within subjects across Sessions 2 and 3 and across Sessions 4 and 5. The order was constant for Sessions 2 and 4 and for
Sessions 3 and 5 so as to address the question of degree of unbalancing appropriately.

Patients were asked to comment on the MAPs' clarity and pleasantness in comparison with their usual MAP. Patients were informed that changes had been made to the C levels in these new MAPs but were not influenced to think that these changes would be disadvantageous.

Patients were not asked to take home the unbalanced MAPs because the aim of the study was not to see whether they could adjust over time to an unnatural coding of frequency information. It also was considered unethical to expect patients to trial unnatural MAPs that may have sounded unpleasant and that were most unlikely to enhance speech perception. After an unbalanced MAP was programmed into the processor, the patients were, however, given a 10 minute adjustment period during which the tester and patient chatted.

Assessments - Phoneme scores were obtained using CNC words (based on 50 word lists published by Peterson and Lehiste [1962]). CUNY sentences were used to assess sentence perception in noise (Boothroyd, Hanin, \& Hnath, Reference Note 1). In the original version of this test, topic words for each of the 12 sentences in a list were provided. In the present study, topic words were omitted and new recordings were made that randomized the topic order of the lists. A signal to noise ratio (SNR) was chosen for the sentence test that ensured that the patient scored in the 50 to $80 \%$ range in the $\mathrm{BC}$ in the second appointment to optimize the test sensitivity (Dillon, 1983). This ratio was constant for all sessions. Table 1 shows the SNRs for each patient. Both perception tests were recorded and played back through a loudspeaker at $65 \mathrm{dBA}$ peak to peak. For the sentence test, eight-talker babble was mixed with the speech at the appropriate levels and SNRs. The SNR was based on the peak dBA level of the speech and the peak $\mathrm{dBA}$ level of the competing babble, each of which were measured on a sound level meter using a fast response. Tests were presented in an audition-alone mode. The order in which the tests were administered was counterbalanced across subjects. Two lists of each test were administered consecutively in each condition for all sessions. Patients never received the same list of sentences or words twice.

Analyses - One-way, repeated measures analyses of variance (ANOVAs) were used to assess whether the group performance in the $20 \%$ UC differed from that in the BC for each of the speech materials. For individual patients, a Chi-squared statistic $(p<$ 0.05 ) with a two-tailed criterion was used to assess whether performance in the $\mathrm{BC}$ was significantly different from performance in the $20 \%$ UC for pho- 
nemes and sentences in noise. Cumulative scores for the $\mathrm{BC}$ for each of the speech materials were derived from four lists-two lists assessed in Session 2 and two lists assessed in Session 3. Similarly, cumulative scores for the $20 \%$ UC were derived from four lists.

In addressing Hypothesis 2 regarding the effect of altering the degree of unbalancing, one-way, repeated measures ANOVAs were used to determine whether group performance in the UC was significantly different from performance in the $\mathrm{BC}$ for each degree of unbalancing; for the LEAST-UC, either 10 or $20 \%$ and the MOST-UC, either 20 or $40 \%$. For example, if a patient performed significantly worse in the $20 \%$ UC, the degree of imbalance was halved to $10 \%$ for the final two sessions. In this case, the $20 \%$ UC was viewed as the MOST-UC and the $10 \%$ UC as the LEAST-UC. An ANOVA comparing the difference scores (BC - UC) for the two different degrees of unbalancing also was computed. In the individual analyses, two Chi-squared results were considered; $\mathrm{BC}$ scores were compared with the UC scores for the LEAST-UC and for the MOST-UC.

One-way ANOVAs for independent groups were used to determine whether the difference scores $(\mathrm{BC}-\mathrm{UC})$ for Multipeak users were significantly different from the difference scores for Speak users.

\section{Results}

\section{$B C$ versus $U C$}

One-way, repeated measures ANOVAs revealed that mean performance in the UC was significantly poorer than that in the $\mathrm{BC}$ for sentences presented in noise and for phonemes $(F[1,9]=8.45 ; p=0.017$ for sentences; $F[1,9]=21.36$, and $F[1,9]=40.07$; $p=0.0001$ for phonemes; see mean scores in Fig. 2 and 3 ). These analyses used mean scores based on four lists in the $\mathrm{BC}$ and four lists in the $20 \% \mathrm{UC}$ for each patient. There was no evidence of a practice effect over time in the $\mathrm{BC}$ when the condition of list order was analyzed. Similarly, a practice effect was not evident in the UC for sentences or phonemes.

Figure 2 shows the individual sentence perception in noise results averaged across four lists for the $\mathrm{BC}$ versus the $20 \%$ UC. A significant decrease in performance in the 20\% UC was evident for 6 of the 10 patients (5 through 10). For Patient 1, however, there was a significant increase in performance in the $20 \%$ UC at the 0.05 level. Four patients $(5,6,7$, and 8) performed significantly poorer in the $20 \% \mathrm{UC}$ for phoneme perception (Fig. 3).

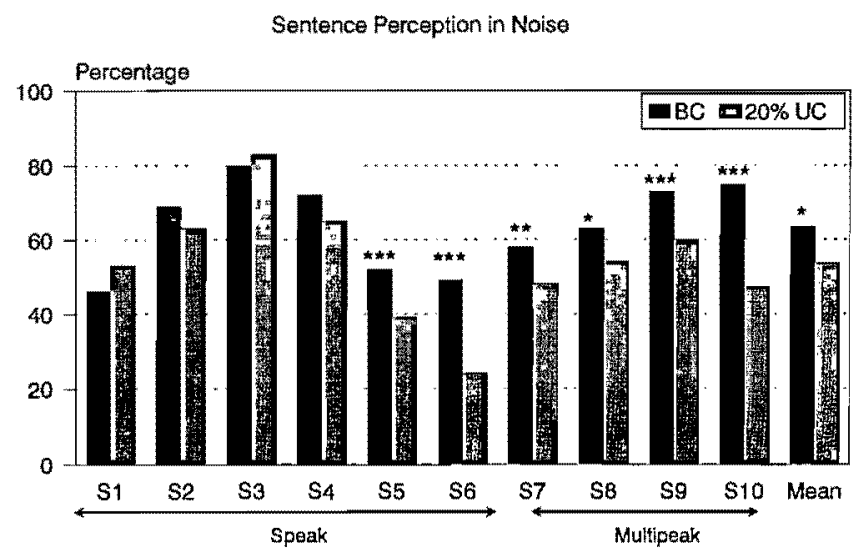

Figure 2. Individual patients' percentage scores and group mean percentage score for sentence perception in noise in the balanced condition ( $\mathrm{BC}$ ) and $20 \%$ unbalanced condition (UC). Significance levels refer to Chi-squared comparisons of the scores in these two conditions. ${ }^{*} p<0.05 ;{ }^{* *} p<0.01$; ${ }^{* * *} p<0.001$. Subjects (S) 1 through 6 used the Speak strategy, and Subjects 7 through 10 used the Multipeak strategy.

\section{Degree of Unbalancing}

The LEAST-UC was either 10\% (Patients 5 through 10 ) or $20 \%$ (Patients 1 through 4), and the MOST-UC was either $20 \%$ (Patients 5 through 10) or 40\% (Patients 1 through 4; see Fig. 4 and 5). The LEAST- and MOST-BC scores were the average scores from four $\mathrm{BC}$ lists conducted in the sessions associated with the LEAST-UC and four BC lists conducted in sessions associated with the MOSTUC, respectively. Table 3 indicates that in the LEAST-UC, group mean performance was not significantly different from performance in the $\mathrm{BC}$ for

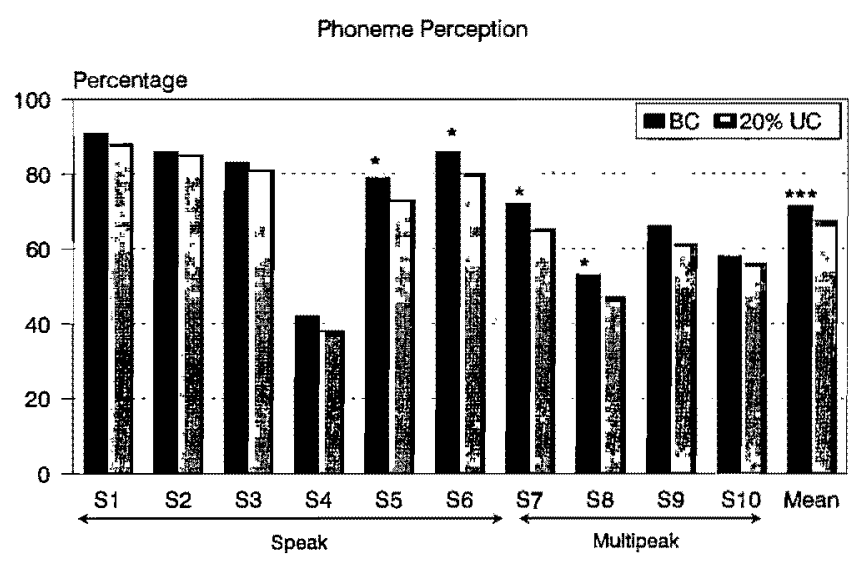

Figure 3. Individual patients' percentage scores and group mean percentage score for phoneme perception in the balanced condition (BC) and $20 \%$ unbalanced condition (UC). Significance levels refer to Chi-squared comparisons of the scores in these two conditions. ${ }^{*} p<0.05 ;{ }^{* *} p<0.01 ;{ }^{* * *} p<$ 0.001. 


\section{Sentence Perception in Noise}
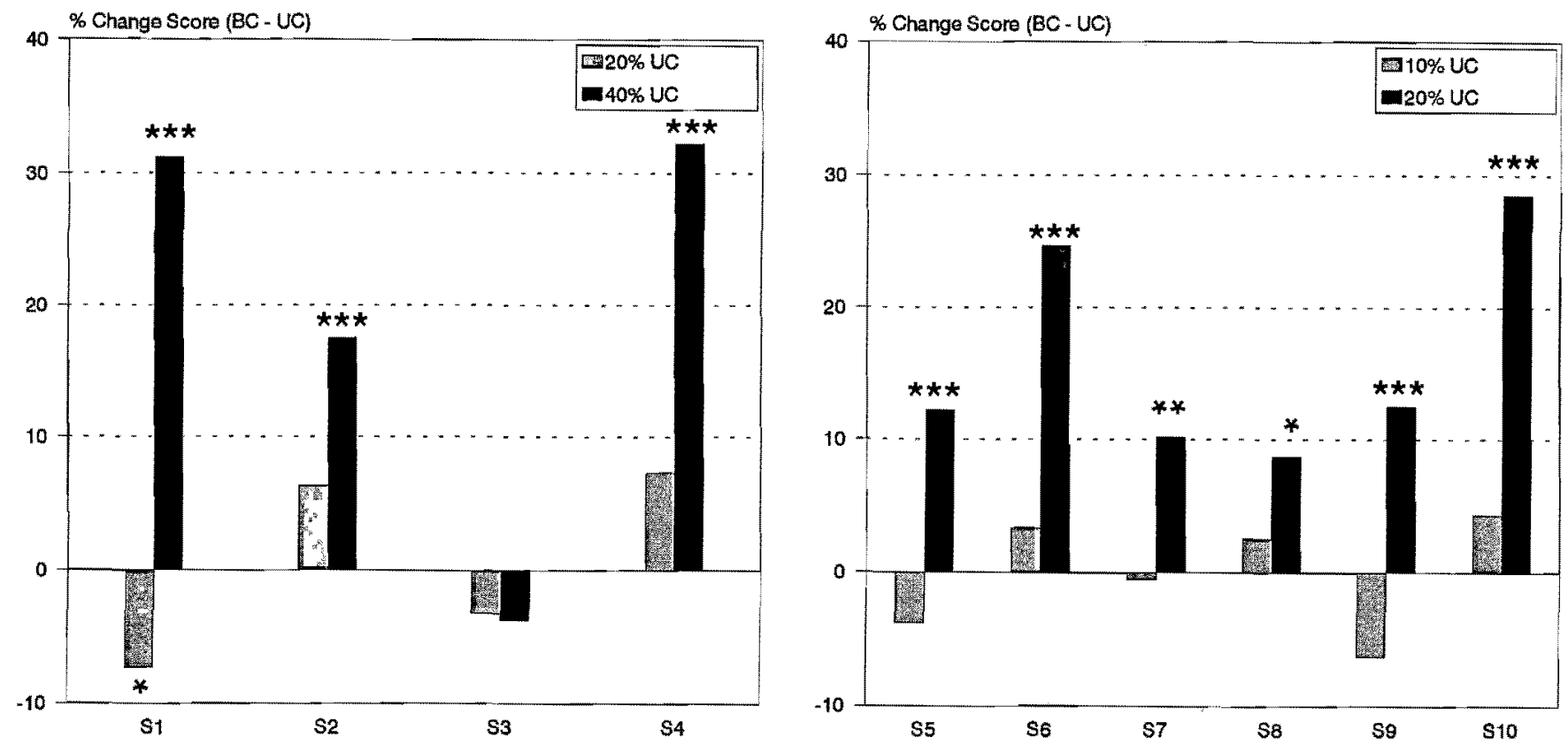

Figure 4. Patients' percentage difference scores $(B C-U C)$ for sentence perception in noise with varying degrees of imbalance between electrodes. ${ }^{*} p<0.05 ;{ }^{* *} p<0.01 ;{ }^{* * *} p<0.001 . \mathrm{BC}=$ balanced condition; $\mathrm{UC}=$ unbalanced condition.

\section{Phoneme Perception}
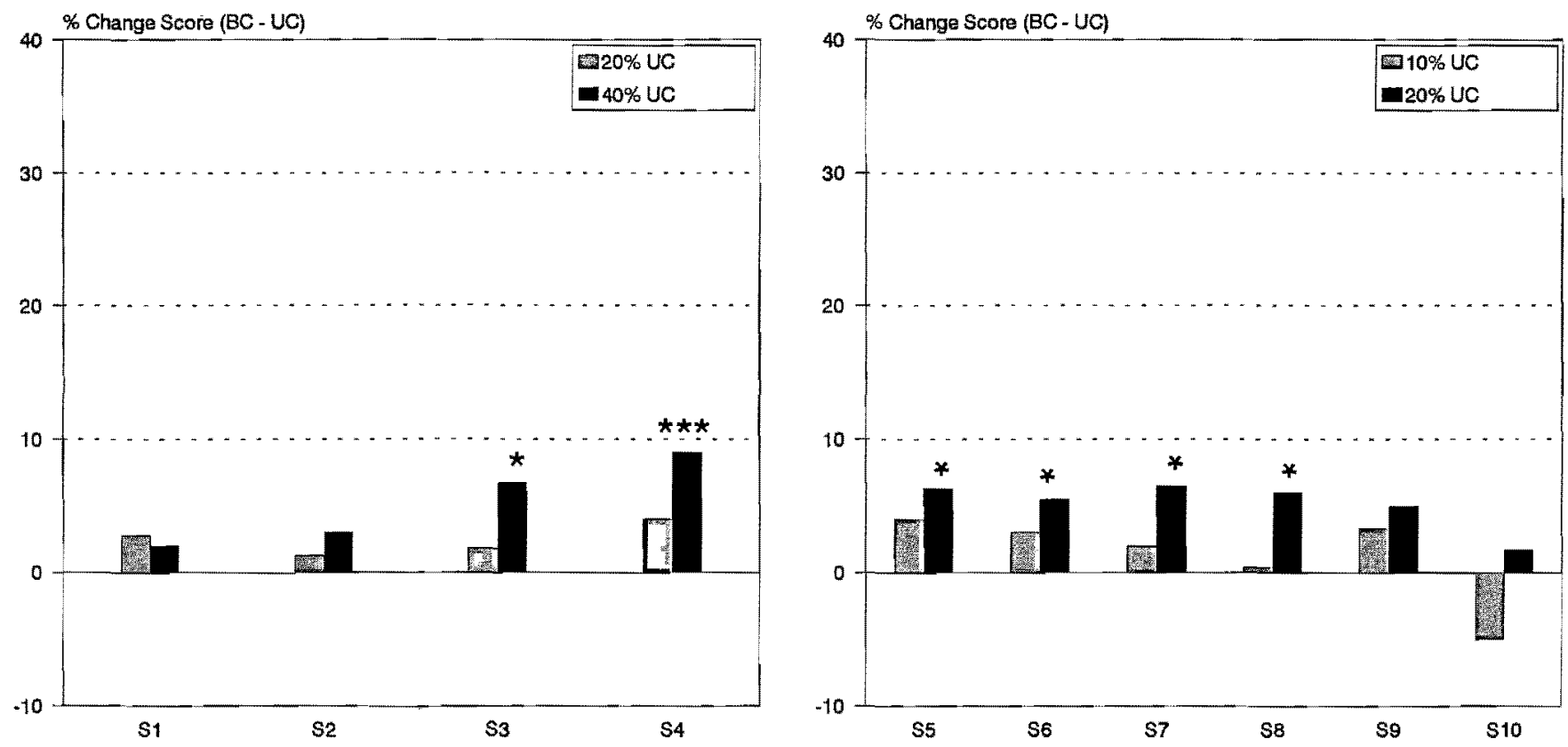

Figure 5. Patients' percentage difference scores (BC - UC) for phoneme perception with varying degrees of imbalance between electrodes. ${ }^{*} p<0.05 ;{ }^{* *} p<0.01 ;{ }^{* *} p<0.001$. $\mathrm{BC}=$ balanced condition; $\mathrm{UC}=$ unbalanced condition.

sentences or phonemes. In contrast, performance was significantly better in the $\mathrm{BC}$ than in the MOST-UC for each of the speech materials. The average difference scores (BC - UC) for the MOST-UC sessions were significantly greater than the difference scores for the LEAST-UC sessions for 
TABLE 3. Group mean percentage scores in the balanced versus unbalanced conditions (BC and $U C)$ for the LEAST-UC and MOST-UC sessions for sentences and phonemes. BC scores marked with superscripts are significantly higher than the MOST-UC scores.

\begin{tabular}{cccccc}
\hline \multirow{2}{*}{$\begin{array}{c}\text { Degree of } \\
\text { Unbalancing }\end{array}$} & \multicolumn{2}{c}{ Sentences } & & \multicolumn{2}{c}{ Phonemes } \\
\cline { 2 - 3 } \cline { 5 - 6 } & $\mathrm{BC}$ & UC & & BC & UC \\
\hline LEAST & 60.1 & 59.9 & & 71.7 & 70.0 \\
MOST & $65.9^{*}$ & 48.5 & & $70.5^{*}$ & 65.3 \\
\hline \hline $0<0.001$. & & & & & \\
\hline
\end{tabular}

sentences in noise and for phonemes $(F[1,9]=32.78$; $p=0.0003$ and $F[1,9]=21.46 ; p=0.001$, respectively; see Fig. 4 and 5 for individual difference scores).

Figure 4 shows the percentage difference scores (BC - UC) in sentence perception for each patient for each degree of imbalance received. These scores are derived from the average of four lists in each condition. Sentence perception in noise in the LEAST-UC was not significantly different from sentence perception in the $\mathrm{BC}$ for any of the 10 patients. This can be seen in the small percentage change scores represented by the light grey bars. In contrast, performance in the MOST-UC was significantly worse than performance in the $\mathrm{BC}$ for 9 of the 10 patients (seen in the larger difference scores represented by the darkened bars). Unbalancing up to $\pm 40 \%$ of the electrodes' DRs did not worsen sentence performance for Subject 3 . Figure 5 shows that phoneme recognition for this patient did, however, worsen significantly with this degree of unbalancing $(p<0.05)$. In keeping with the sentence results, no patient showed significantly poorer performance in the LEAST-UC for phoneme perception (indicated by small change scores). Six of the 10 patients $(3,4,5,6,7$, and 8$)$ showed a significant decrease in performance in the MOST-UC (indicated by higher change scores).

It can be assumed that patients who performed worse in the $20 \%$ UC also would have performed worse in a $40 \%$ UC and that patients who did not perform worse in the $20 \%$ UC also would not have performed worse in a $10 \%$ UC. From these assumptions it can be interpolated that for phoneme perception, no patients showed a significant drop in performance in the $10 \% \mathrm{UC}, 4$ of the 10 patients did worse in the $20 \% \mathrm{UC}$, and 6 of the 10 patients did worse in the $40 \%$ UC. Similarly, it can be interpolated that for sentence perception in noise, no patients did worse in the $10 \%$ UC, 6 of the 10 patients performed worse in the $20 \%$ UC and 9 of the 10 patients did worse in the $40 \% \mathrm{UC}$.

\section{Comparison of Multipeak and Speak Strategies}

For sentence perception in noise, all four users of the Multipeak strategy performed significantly worse with $20 \%$ unbalancing, whereas only two of the six patients using the Speak strategy performed worse with this amount of unbalancing (see Fig. 2). Despite this, the difference scores $(\mathrm{BC}-\mathrm{UC})$ for Multipeak users were not significantly greater than the difference scores for Speak users for either of the speech materials assessed.

\section{Subjective versus Objective Evaluations}

All of the patients reported a qualitative difference in the sound in the $20 \%$ UC. Typical comments regarding the quality of the sound included: it sounds rough; it echoes; some sounds are very dominant, boom out and hiss. Half of the patients $(1,2$, 5,6 , and 9) described the difference in clarity and quality of sound as minimal. The latter three patients showed a significant drop in perception. Of those patients who described the $20 \%$ UC as definitely worse in clarity and quality $(4,7,8$, and 10$)$, Patient 4 showed no significant decrease in speech perception. Patient 3 , whose speech perception was unaffected in the $20 \% \mathrm{UC}$, described the $20 \%$ unbalanced MAPs as less pleasant but equally as clear as the balanced MAP. Six patients were then given MAPs with only $10 \%$ unbalancing. Speech perception was not affected with this degree of unbalancing for all patients. Four of these patients $(5,6,8$, and 9$)$ perceived a minimal difference, commenting that the sound was as good as or better than their balanced MAP. Two patients (7 and 10) reported that the unbalanced MAPs were worse in quality and clarity.

Four subjects ( 1 through 4 ) were given MAPs with $40 \%$ unbalancing. All patients showed a drop in perception for at least one of the speech materials assessed and reported poorer quality of sound. $\mathrm{Pa}$ tients 2 and 3 reported little to no change in clarity for these unbalanced MAPs.

\section{Discussion}

\section{$B C$ versus $\mathrm{UC}$}

Group results revealed that performance in the $20 \%$ UC was significantly worse than that in the BC for each of the speech materials assessed. Individual analyses indicated that 6 of the 10 patients scored significantly worse for sentence material in noise in the $20 \%$ UC than in the BC. Four of these six patients also performed worse for phonemes.

These results confirm anecdotal evidence from clinicians that an unbalanced MAP can adversely 
affect performance. For two of the six patients ( 9 and 10) who performed worse in the $20 \% \mathrm{UC}$, changes to the $\mathrm{C}$ levels often were five stimulus levels or less (changes to $\mathrm{C}$ levels for Patient 9 are shown in Fig. 1). Each of these patients had narrow DRs (see Table 1). In contrast, the performance of Patient 2 , who also had a relatively small $D R$ and relatively small changes to $\mathrm{C}$ levels, was not degraded in the $20 \%$ UC. Despite this, the results for Patients 9 and 10 confirm the need to be conservative and use an increment less than five stimulus levels when mapping $\mathrm{C}$ levels. It is likely that the lowering of many electrodes' C levels in the MAPs with $20 \%$ imbalance weakened the clarity of the spectral cues for some speech sounds. Louder phonemes (60 to $70 \mathrm{~dB}$ SPL) that stimulate the top $20 \%$ of the DR would be more affected by the unbalancing than would softer phonemes. In the present study, the effect of making some speech sounds softer with the lowering of $\mathrm{C}$ levels was not separated out from the effect of imbalance in the loudness of the $C$ levels across the electrode array. Perhaps these effects could be better controlled in further research that, for example, compares the effect of a MAP with a $20 \%$ drop in C levels for all electrodes with a MAP that has a $20 \%$ drop in the $\mathrm{C}$ level for every alternate electrode.

\section{Degree of Unbalancing}

Sentence perception in noise and word and phoneme perception for the group was significantly worse when the degree of unbalancing was doubled, either from $10 \%$ to $20 \%$ or from $20 \%$ to $40 \%$ (see Table 3). This finding was supported by the individual sentence and phoneme perception results, which showed that 9 of the 10 patients and 6 of the 10 patients, respectively, were adversely affected in the MOST-UC. No patient performed worse relative to the BC in the LEAST-UC. For some individuals, there was notable variability in the $\mathrm{BC}$ scores across time from the sessions associated with the LEAST-UC to the sessions associated with the MOST-UC. This is reflected in the difference between the average $\mathrm{BC}$ scores associated with the MOST-UC and the BC scores associated with the LEAST-UC sessions (Table 3). Changes in the patient's mood, health, attentiveness, and ability to do the task may have occurred across sessions and contributed to these differences. The analysis in this study focused on differences between the BC and UC within sessions, when it can be assumed that such factors are more constant. Counterbalancing of the order in which the conditions were assessed helped to reduce the effects of fatigue or practice within a session.

An interesting finding was that in the $20 \% \mathrm{UC}$, no patient suggested a desire to turn the sensitivity control down, although MAPs often were described as echoing and fluctuating in loudness. Only one of the four patients (Patient 4) who received MAPs with $40 \%$ unbalancing opted to turn down the sensitivity control, and even in this instance the change was only half a level (which corresponds to a decrease of approximately $3 \mathrm{~dB}$ ). Of course the patients may have been less tolerant in real-life situations over an extended period of time. This finding does, however, suggest that children may not reject very randomly unbalanced MAPs by refusing to wear the device or by turning the sensitivity control down to a very low level.

\section{Differences in Individual Performance}

Individual differences in performance with unbalancing of the $\mathrm{C}$ levels could be attributable to a number of factors including the SNR used in the sentence testing, place pitch perception, and which particular electrodes were predominantly unbalanced.

One could hypothesize that unbalancing the $\mathrm{C}$ levels will have a worse effect on speech perception (relative to the $\mathrm{BC}$ ) when the SNR is lower and there is a greater spread of activity across the electrode array. Unbalancing of the $\mathrm{C}$ levels can weaken spectral information. This effect of unbalancing may be more detrimental when the spectral information has already been weakened to a considerable extent by the presence of noise. When spectral information is poor, patients may rely more on amplitude information. The natural amplitude cues of speech sounds are disrupted in the UC. For this group of patients, there was no indication that lower SNRs were associated with poorer performance in the $20 \%$ $\mathrm{UC}$ relative to the $\mathrm{BC}(r=0.288 ; p=0.42)$. This finding should be treated with caution, though, given the small size of the group and the fact that other variables, such as the extent of unbalancing for particular electrodes and the processing strategy used, were not constant across individual subjects. Patients using the Speak strategy generally had lower SNRs, which reflected their superior sentence perception in background noise.

The ability to use spectral cues also could be a factor affecting individual performance in the UC. The patients with an excellent ability to use frequency or place pitch information may be unperturbed by deviant amplitude information. Other patients with inferior place pitch skills may need to rely to some extent on amplitude cues. This could be explored in further research with synthesized or computer-controlled speech in which frequency cues are preserved while amplitude cues are either equal- 
ized or distorted in a controlled manner. An analysis could then be done to determine whether this estimate of a patient's place pitch ability is correlated with performance in the UC.

Individual differences in performance in the $20 \%$ UC may have been influenced by the fact that each patient had different unbalanced MAPs derived from different sets of random numbers (see Methods). A constant set of random numbers could not have been used for each patient because different numbers of electrodes were in some patients' MAPs. Consequently, the percentage change to the $C$ levels for particular frequency ranges differed across individuals. It is conceivable that an unbalanced MAP would be more detrimental if there were substantial drops in the $\mathrm{C}$ levels for electrodes that were being stimulated more frequently during the speech lists.

It is possible that individual differences in performance also may be attributable in part to the fact that some patients are more distracted than others by any change to their familiar MAP. Performance with unbalanced MAPs may have improved if patients had had some take-home experience with these MAPS.

\section{Comparison of Multipeak and Speak Strategies}

On average, Multipeak users did not seem to be more adversely affected by unbalancing of $\mathrm{C}$ levels than did the Speak users. There was a difference in the expected direction for sentence perception in noise, but the difference scores (BC - UC) for Multipeak users were not significantly higher than those for Speak users.

Skinner et al. (1994) found improved perceptual performance with the Speak strategy compared with the Multipeak strategy, especially for speech material presented in noise. Spectral peaks of speech sounds are less conspicuous in noise. Unbalancing of the $C$ levels can further weaken spectral information by making some speech sounds less salient. Furthermore, when spectral cues are poorer, patients may need to rely more on temporal amplitude information. The UC upsets the natural amplitude cues of speech sounds. It is possible that unbalancing $\mathrm{C}$ levels may have a more adverse effect on speech perception when spectral information already has been weakened by noise. It was therefore anticipated that the users of the Speak strategy would be less disadvantaged in the UC than would the Multipeak users, especially for sentence perception in noise. Only one electrode is stimulated at a time for the first and second formants with the Multipeak strategy, and in the UC there may be at times substantial changes to the $C$ levels of the electrodes selected. In contrast, several electrodes can be stimulated for a single formant with the Speak strategy, and some of these may have little to no change to the $C$ levels. The absence of change for some electrodes may offset the large changes on other electrodes stimulated. This redundancy of for: mant information with the Speak strategy especially facilitates perception in noise. In contrast, spectral peaks for speech may be shifted in the presence of noise with the feature extraction Multipeak strategy.

A significant difference between Multipeak and Speak users may have occurred with a larger group of patients. Furthermore, the present findings are compromised by the fact that two groups of patients were not matched on other factors such as the SNR used in the testing and the particular unbalanced MAPs received. The Speak users generally had lower SNRs, which is not surprising given the findings of superior performance for the Speak strategy in noise (Skinner et al., 1994).

\section{Subjective versus Objective Evaluations}

Patients' qualitative comments regarding the unbalanced MAPs were not closely related to their speech perception results. In some cases, patients reported minimal differences but a significant result occurred for perception. In other cases, no decrease in perception occurred but patients reported that the unbalanced MAPs were worse in quality and/or clarity. Patients described the effects of unbalancing similarly with phrases such as "it sounds rough, it echoes." This study's findings suggest the importance of considering both types of evaluation in fine tuning a patient's MAP. It was anticipated that patients' comments on speech clarity or intelligibility would be reasonably compatible with speech perception performance. In contrast, comments on "pleasantness" or speech "quality" were expected to be less related to perception scores. This was not the case because for all patients except Patient 3 , comments on the clarity of sound were in line with comments on the sound quality.

\section{Conclusions}

The $\mathrm{C}$ levels in the MAPs of 10 adults implanted with the 22-electrode cochlear prosthesis were randomly adjusted up and down between 0 and $\pm 20 \%$ of each electrodes' DR. Group mean scores for tests assessing sentence perception in noise and phoneme perception in words in quiet were poorer in this UC than in the $\mathrm{BC}$. The majority of patients showed poorer performance for sentences in noise with $20 \%$ unbalancing. Although statistically significant, the changes $(\mathrm{BC}-\mathrm{UC})$ were relatively small for several 
patients (for four of the six patients, changes ranged from 9 to $13 \%$ ). Changes to the phoneme scores were less than $10 \%$. For the four patients who showed no initial decline in perception, the degree of unbalancing was doubled and a significant difference then occurred between the $\mathrm{BC}$ and the UC for sentence and/or phoneme scores. For the remaining six patients, it was found that $10 \%$ of unbalancing, which usually changed stimulus levels by less than five levels, had no detrimental effect on perception.

It is possible that many children younger than 5 yr of age have unbalanced $\mathrm{C}$ levels in their MAPs, which in conjunction with other factors may prevent them from fulfilling their potential with the implant. If a young child's communication development and speech perception performance with the implant is poor, clinicians should be aware that loudness imbalance between electrodes could be contributing to this lack of progress. It is also possible, however, that children learn to adapt to unbalanced MAPs over time. This study did not address the possibility that unbalanced MAPs may have a less detrimental effect on speech perception if patients are given a longer period of time to adjust to them.

Further research could attempt to explore why some patients are more affected by unbalancing than are others and determine the relative importance of influences such as softening the signal for some speech sounds and the disruption of temporal amplitude cues.

\section{AckNowledgments:}

The authors gratefully acknowledge the financial support of the Co-operative Research Centre for Cochlear Implant, Speech and Hearing Research, and the Australian Bionic Ear and Hearing Research Institute. We also express gratitude to the adults participating in the study. Many thanks to Dr. Peter Blamey, Dr. Hugh McDermott, Dr. Peter Busby, Dr. Colette McKay, and Leslie Whitford for their constructive comments in the design and analysis of this research.

Address for correspondence: Ms P. Dawson, The Australian Bionic Ear and Hearing Research Institute, 384-388 Albert St, East Melbourne, Victoria 3002, Australia.

Received November 11, 1995; accepted August 14, 1996

\section{ReferenCes}

Battmer, R-D., Laszig, R., \& Lehnhardt, E. (1990). Electrically elicited stapedius reflex in cochlear implant patients. Ear and Hearing, 11, 370-374.

Berger, K., Hagberg, N. S., \& Rane, R. L. (1977). Prescription of hearing aids. Kent, OH: Herald Publishing Co.

Blamey, P. J., Dowell, R. C., \& Clark, G. M. (1987). Acoustic parameters measured by a formant-estimating speech proces- sor for a multiple-channel cochlear implant. Journal of the Acoustical Society of America, 82, 38-47.

Byrne, D., \& Tonisson, W. (1976). Selecting the gain of hearing aids for persons with sensorineural hearing impairments. Scandinavian Journal of Audiology, 5, 51-59.

Clark, G. M., Blamey, P. J., Busby, P. A., Dowell R. C., Franz, B. K.-H, Musgrave, G. H., Nienhuys, T. G., Pyman, B. C., Roberts, S. A., Tong, Y. C., Webb, R. L., Kuzma, J. A., Money, D. K., Patrick, J. F, \& Seligman, P. M. (1987). A multiple-electrode cochlear implant for children. Archives of Otolaryngology Head and Neek Surgery, 113, 825-828.

Dillon, H. (1983). The effect of test difficulty on the sensitivity of speech discrimination tests. Journal of the Acoustical Society of America, 73, 336-344.

Peterson, G., \& Lehiste, I. (1962), Revised CNC lists for auditory tests. Journal of Speech and Hearing Disorders, 27, 62-70.

Ptacek, P. H., \& Koutstaal, C. W. (1977). Identification of vowels with equated intensity. A preliminary study. Journal of the American Audiology Society, 2, 169-172.

Shallop, J. K. (1993). Objective electrophysiological measures from cochlear implant patients. Ear and Hearing, 14, 58-63.

Shallop, J. K., VanDyke, L., Goin, D. W., \& Mischke, R. E. (1991). Prediction of behavioural threshold and comfort values for Nucleus 22-channel implant patients from electrical auditory brain stem response test results. Annals of Otology Rhinology and Laryngology, 100, 896-898.

Skinner, M. W., Clark, G. M., Whitford, L. A., Seligman, P. M., Staller, S. J., Shipp, D. B., Shallop, J. K., Everingham, C., Menapace, C. M., Arndt, P. L., Antognelli, T., Brimacombe, J. A., Pijl, S., Daniels, P., George, C. R., McDermott, H. J., \& Beiter, A. L. (1994). Evaluation of a new spectral peak coding strategy for the nucleus 22 channel cochlear implant system. American Journal of Otology, 15(Suppl. 2), 15-27.

Skinner, M. W., Holden, L. K., Holden, T. A., Dowell, R. C., Seligman, P. M., Brimacombe, J. A., \& Beiter, A. L. (1991). Performance of postlinguistically deaf adults with the wearable speech processor (WSP III) and mini speech processor (MSP) of the Nucleus multi-electrode cochlear implant. Ear and Hearing, 12, 3-22.

Skinner, M. W., Pascoe, D. P., Miller, J. D., \& Poelka, G. R. (1981). Measurements to determine the optimal placement of speech energy within the listener's auditory area: a basis for selecting amplification characteristics. In G.A. Studebaker \& F.H. Bess (Eds.), The Vanderbilt Report; State Art-Research Needs. Monographs in Contemporary Audiology. Upper Darby, PA: Instrumentation Associates.

Spivak, L. G., \& Chute, P. M. (1994). The relationship between electrical acoustic reflex thresholds and behavioral comfort levels in children and adult cochlear implant patients. Ear \& Hearing, 15, 184-192.

Staller, S. J., Beiter, A. L., \& Brimacombe, J. A. (1994). Use of the Nucleus 22 channel cochlear implant system with children. The Volta Review, 96 (5), 15-39.

Van Tasell, D. J., Soli, S. D., Kirby, V. M., \& Widon, G. P. (1987). Speech waveform envelope cues for consonant recognition. Journal of the Acoustical Society of America, 82, 1152-1161.

\section{REFERENCE Notes}

1 Boothroyd, A., Hanin, L., \& Hnath, T. (1985). A sentence test of speech perception: reliability, set equivalence, and short term learning. Internal report, Speech \& Hearing Sciences Research Center, City University of New York. 


\section{University Library}

\section{- M M I N E R VA A gateway to Melbourne's research publications}

Minerva Access is the Institutional Repository of The University of Melbourne

\section{Author/s:}

Dawson, P. W.;Skok, M.;Clark, Graeme M.

Title:

The effect of loudness imbalance between electrodes in cochlear implant users

Date:

1997

Citation:

Dawson, P. W., Skok, M., \& Clark, G. M. (1997). The effect of loudness imbalance between electrodes in cochlear implant users. Ear and Hearing, 18(2), 156-165.

Persistent Link:

http://hdl.handle.net/11343/27555 